\title{
Microencapsulation of citronella oil by complex coacervation using chitosan-gelatin (b) system: operating design, preparation and characterization
}

\author{
Fitrah Rabani Abdul Aziz, Junaidah Jai, Rafeqah Raslan and Istikamah Subuki \\ Faculty of Chemical Engineering, Universiti Teknologi MARA, 40450 Shah Alam, Selangor, Malaysia
}

\begin{abstract}
Citronella oil (CO) can be an effective mosquito repellent, but due to its nature which having high volatility, oils rapidly evaporates causing loss of efficacy and shorten the repellent effect. Therefore, microencapsulation technology was implemented to ensure the encapsulated material being protected from immediate contact with environment and offers controlled release. In this study, microencapsulation of $\mathrm{CO}$ was done by employing complex coacervation using chitosan-gelatin (B) system and utilized proanthocyanidins as the crosslinker. Remarkably, nearly all material involved in this study are from natural sources which are safe to human and environment. In designing operating process condition for $\mathrm{CO}$ encapsulation process, we found that wall ratio of $1: 35$ and $\mathrm{pH} 5$ was the best operating condition based on zeta potential and turbidity analysis. FT-IR analysis found that gelatin-B had coated the $\mathrm{CO}$ droplet during emulsification stage, chitosan started to interact with gelatin-B to form a polyelectrolyte complex in adjust $\mathrm{pH}$ stage, $\mathrm{CO}$ capsules solidified at cooling process and were hardened during crosslinking process. Final product of CO capsules after settling process was identified at the top layer. Surface morphology of CO capsules obtained in this study were described having diameter varies from $81.63 \mu \mathrm{m}$ to $156.74 \mu \mathrm{m}$ with almost spherical in shape.
\end{abstract}

\section{Introduction}

Citronella oil $(\mathrm{CO})$ is an essential oils extracted from aromatic grasses of Citronella grass. It is classified in trade into two types; Ceylon $\mathrm{CO}$, obtained from Cymbopogon nardus (inferior type), while Java CO obtained from Cymbopogon winterianus (superior type). This plant can be found growing wild in most tropical Asian countries including Malaysia. CO is comparatively high demand due to its usage in traditional medication. It also widely used in perfume industry, soap manufacturing, cosmetics, flavouring industry and health purposes [1]. In addition, $\mathrm{CO}$ also shows good potential usage in food packaging and textile industry $[2,3]$. In fact, Environmental Protection Agency (EPA) itself has classified $\mathrm{CO}$ as a biopesticide with a non-toxic mode of action $[4,5]$. Therefore, $\mathrm{CO}$ can be found in dozens of registered pesticide products such as sprays, lotions, candles, sunscreen, wristbands, and pet's collars $[4,6]$. This makes essential oil (EO) from Cympobogon species such $\mathrm{CO}$ is popular in insect or mosquito repellent formulations. Candles and incense containing $\mathrm{CO}$ are sold as insect repellents in some countries like the United States [7]. There was also a study done by [8] which utilized $\mathrm{CO}$ in formulation of mosquito repellent waterbased paint. It was suggested by [9] and [10] that $\mathrm{CO}$ demonstrates good efficacy against mosquitoes in concentrations ranging from $0.05 \%$ to $15 \%(\mathrm{w} / \mathrm{v})$ alone or in combination with other natural or commercial insect repellent products. Although synthetic chemical $N, N$ diethyl-3-methylbenzamide (DEET) is currently the most effective mosquito repellent, but due to adverse effects reported such include skin eruption in children and irreversible damage to the ecosystem from nondegradable compound in DEET, development of effective non-DEET alternatives is needed [7]. Thus, citronella is one of the most widely used natural repellents on the market nowadays and was used at concentrations of 5-10\%. It was stated in [11] that most of the EO-based repellents tend to give short lasting protection for less than 2 hours. However, $\mathrm{CO}$ was found to give 2 hours repellency and be the most effective mosquito repellent compared to the other thirty eight EOs that have been studied in [11]. Initially, $\mathrm{CO}$ can be as effective as DEET in repels mosquito or insect, but due to its nature, oils rapidly evaporate causing loss of efficacy and leaving the user unprotected [12].

Microencapsulation technology is well known as a process of packaging micronized liquid or solid material in the form of microcapsules. This process give the encapsulated material affirm protection from immediate contact with environment besides provide controlled release [13]. Numerous manufacturing approaches have been adopted in producing microcapsules and the most common used processes include complex coacervation, spray drying, centrifugal extrusion, air suspension coating, 
pan coating, emulsion hardening process, polymerpolymer incompatibility, interfacial polymerization and in-situ polymerization $[14,15]$. By comparing with various microencapsulation techniques, [16] claimed that coacervation is one of the simplest and cheapest ways to form microcapsules. It is a promising technique due to high payloads achievable (up to 99\%) [17]. Complex coacervation simply can be described as method where two or more molecules type of opposite charge, are joined as the walls and then disperse the core substance into the aqueous solution of wall materials. When the conditions are right, the wall materials will attract with each other because of the opposite charges, and then embed the core material. It is widely used to prepare microcapsules for controllable drug release because of its simplicity and low pollution [18].

Systems containing both cationic and anionic hydrophilic colloids can induce coacervation. Thus, complex coacervation is expected to occur between chitosan, a water soluble cationic polysaccharide which is soluble in dilute acids at $\mathrm{pH} 5-6$, and type- $\mathrm{B}$ gelatin, a protein which is negatively charged at $\mathrm{pH}$ values above its isoelectric point which is around $\mathrm{pH}$ 4.7-5.2 [19]. Work done by [20] claimed that water stability of chitosan-gelatin (B) blends was found to decrease with greater gelatin content, thus chemical cross-linking is necessary. Cross-linking is a method used to branch chemical and physical junctions within polymeric materials [21]. The most common cross-linker used associates to gelatin are formaldehyde and glutaraldehyde $[21,22]$. Other material that had also been used as crosslinker in chitosan-gelatin system are polyethylene oxide (PEO), polyvinyl acetates (PVA) and 1-ethyl-3-(3dimethylaminopropyl)-carbodiimide (EDC) [22]. However, this kind of synthetic cross-linker unfortunately can cause physiological toxicity [23]. Therefore, natural and nontoxic cross-linker such as Proanthocyanidins (PA) is preferable in order to strengthen and stabilize chitosangelatin blend. This material which majorly contain in fruits and vegetables is about 120 times less toxic than glutaraldehyde [22].

The objectives of this paper can be divided into four: (1) to design operating condition for complex coacervation of $\mathrm{CO}$ using chitosan-gelatin(B) system; (2) to encapsulate $\mathrm{CO}$ using this method; (3) to investigate any changes in chemical structure of material throughout each stage of production process; (4) to analyze surface morphology of prepared CO capsules.

\section{Methodology}

\subsection{Material}

Citronella oil (Java, pure essential oil) was purchased from BF1 (Malaysia). Gelatin-B (type B, 260 bloom, from bovine) was supplied from Halagel Sdn. Bhd. (Malaysia). Chitosan (70-97\% deacetylation, 10-1500 $\mathrm{mPa} . \mathrm{s}$ ) from R\&M Chemicals (Malaysia) was used in this experiment. Oligoproanthocyanidin from grape seed extract (GSE-OPC) contains approximately 80\% Proanthocyanidin was purchased from Kordel's
(Malaysia). Other chemicals in analytical grade such as glacial acetic acid $\left(\mathrm{CH}_{3} \mathrm{COOH}\right)$, sodium hydroxide $(\mathrm{NaOH})$ and de-ionized water (DIW) were used throughout this experiment.

\subsection{Designing wall ratio and operating $\mathrm{pH}$}

Both wall material solutions were prepared according to [24], where solutions of $1 \%(\mathrm{w} / \mathrm{v})$ gelatin-B (Gel-B) solution were prepared by 30 minutes soaking the protein in DIW prior to 1 hour stirring at $50^{\circ} \mathrm{C}$ until fully dissolved. Whereas $1 \%(\mathrm{w} / \mathrm{v})$ chitosan (Chi) solutions were prepared by dissolving analogous amount of Chi in $1 \%(\mathrm{v} / \mathrm{v}) \mathrm{CH}_{3} \mathrm{COOH}$ aqueous solution for more than 12 hours at room temperature. Zetasizer ${ }^{\circledR}$ Nano Series (Malvern Instruments Ltd., Worcestershire, UK) was used to measure zeta potential value of both polymeric solutions at $\mathrm{pH}$ range from 4.0 to 5.6 with 0.2 increments. In order to determine operating $\mathrm{pH}$ and wall ratio, turbidity analysis of wall mixture were conducted. Series of wall ratio was developed from zeta potential data. In a small amount, the wall material solutions were mixed according to the developed ratio and been stirred at $50^{\circ} \mathrm{C}$ $(500 \mathrm{rpm})$ for 30 minutes. The mixed sample solution was then analyzed using spectrophotometers (UviLine 9400, Secomam, France) at $600 \mathrm{~nm}$ wavelength as according to [25].

\section{$2.3 \mathrm{CO}$ encapsulation process}

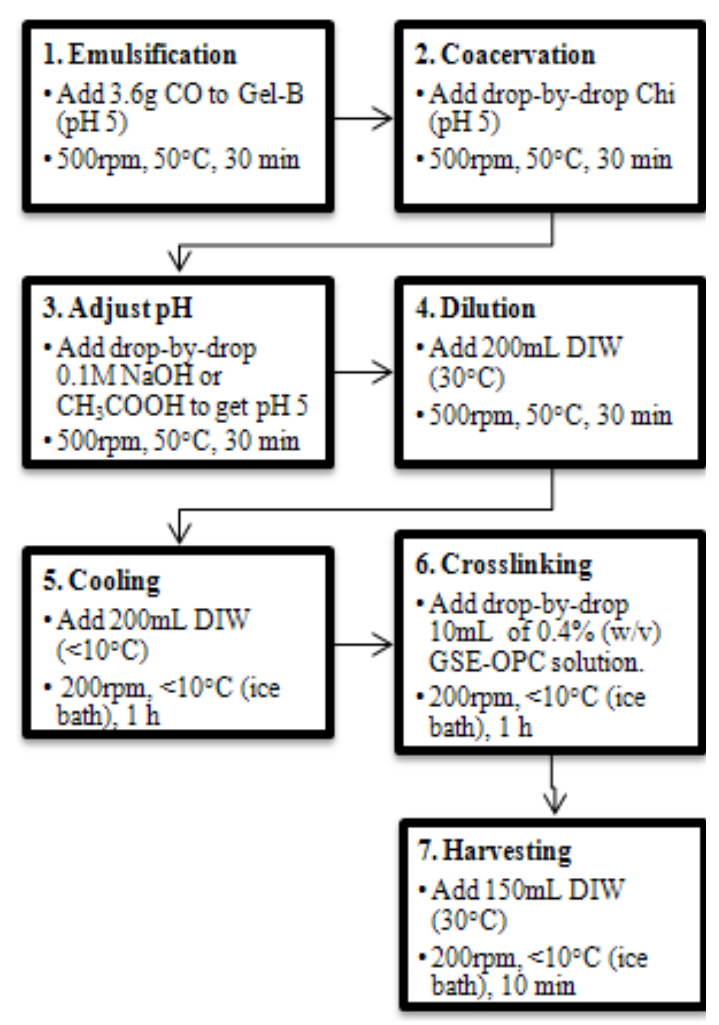

Figure 1. Citronella oil (CO) encapsulation process.

Operating condition for $\mathrm{CO}$ encapsulation process by complex coacervation using Chitosan-Gelatin(B) system was shown in Fig. 1. This study employed $350 \mathrm{~mL}$ Gel-B 
and $10 \mathrm{~mL}$ Chi respectively. The process steps were established by taking [16, 22], [26]-[28] as references. Few drop of mixture from each process step was analyzed by using optical microscope (RZ-5, Meiji Techno, Japan) and Fourier Transform Infra-Red, FT-IR (Perkin Elmer / Spectrum One). The microscopic images were taken at 30x magnification whereas FT-IR spectra were recorded by absorbance (A) mode in wavelength range $4000-515 \mathrm{~cm}^{-1}$.

\subsection{CO capsules product collection and analysis}

After harvesting process, the solution was transferred into a separating funnel and undergoes settling process for more than 12 hours. Three layers were obtained which top layer (contained $\mathrm{CO}$ capsules in super dilute excess polymer mixture), middle layer (contained dilute excess polymer mixture) and bottom layer (contained concentrate excess polymer mixture). Aliquots for each layer were taken for analysis using optical microscope and FT-IR. The bottom layer and middle layer were drained off and the top layer was rinsed with distilled water 3 times in order to get final product of $\mathrm{CO}$ capsules.

\section{Result and discussion}

\subsection{Zeta potential and turbidity analysis}

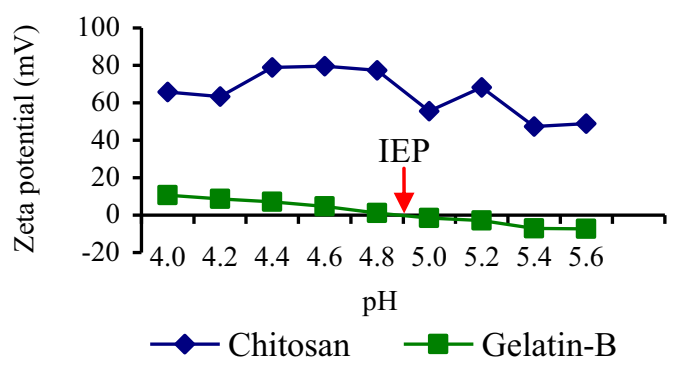

Figure 2. Zeta potential values of $1 \%(\mathrm{w} / \mathrm{v})$ Chitosan and Gelatin-B respected to $\mathrm{pH}$.

In designing operating condition for complex coacervation process, it is essential to know the charge for both of the wall materials. Thus, Fig. 2 shows zeta potential values of $1 \%(\mathrm{w} / \mathrm{v}) \mathrm{Chi}$ and Gel-B respected to $\mathrm{pH}(\mathrm{p}<0.05)$. Based on this data, we observed that all zeta potential values for $\mathrm{Chi}$ are in positive value which indicates that chitosan is positively charge and acts as polycation within range of $\mathrm{pH} 4.0$ to 5.6. This result aligned with [29] which claimed that chitosan being a cationic polysaccharide in neutral or basic $\mathrm{pH}$ conditions but insoluble in aqueous media. However, in acidic condition, chitosan is soluble in water due to protonation of amino groups. Meanwhile, zeta potential values for Gel-B shows both positive and negative value respected to $\mathrm{pH}$. This result signified that gelatin-B having amphoteric properties which its solution can be either positive or negative charge depending on $\mathrm{pH}$. This statement was supported in [30] which claimed that gelatin in general is a polyampholyte obtained from denatured collagen. In this study, the isoelectric point (IEP) which indicates the neutral charge [23] for gelatin was figured out at approximately $\mathrm{pH} 4.9$. As according to [31], in order for complex coacervation takes place to produce capsule, oppositely charged polyelectrolyte are present and mixed in aqueous media causing spontaneous liquid-liquid phase separation. Therefore, the designed operating $\mathrm{pH}$ was restricted between $\mathrm{pH} 5.0$ to 5.6 so that both Chi and Gel-B are able to present as polycation and polyanion respectively.

Table 1. Wall ratio value from ratio of Chitosan (Chi) and Gelatin-B (Gel-B) approximate equal charge

\begin{tabular}{|c|c|c|c|c|c|}
\hline $\mathrm{pH}$ & Chi $(\mathrm{mV})$ & $\begin{array}{c}\text { Gel-B } \\
(\mathrm{mV})\end{array}$ & $\begin{array}{c}\text { Gel-B } \\
\text { approximate } \\
\text { equal charge } \\
(\mathrm{mV})\end{array}$ & Multiplier & $\begin{array}{c}\text { Chi:Gel } \\
-\mathrm{B}\end{array}$ \\
\hline 5.0 & 55.53 & -1.60 & -55.52 & $34.70 \approx 35$ & $1: 35$ \\
\hline 5.2 & 68.23 & -3.00 & -68.22 & $22.74 \approx 23$ & $1: 23$ \\
\hline 5.4 & 47.23 & -7.22 & -47.22 & $6.54 \approx 7$ & $1: 7$ \\
\hline 5.6 & 49.00 & -7.33 & -48.96 & $6.68 \approx 7$ & $1: 7$ \\
\hline
\end{tabular}

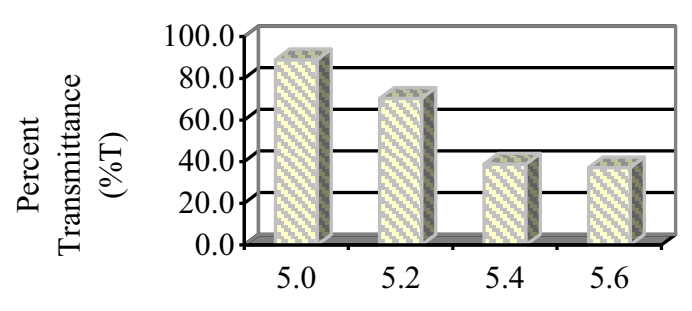

$\mathrm{pH}$

Figure 3. Percent transmittance of Chitosan-Gelatin (B) mixture against $\mathrm{pH}$.

Series of wall ratio for $\mathrm{pH}$ 5.0, 5.2, 5.4 and 5.6 were developed by considering on zeta potential value of both Chi and Gel-B. By using multiplier, approximate equal charge of Gel-B were obtained and wall ratio from $\mathrm{pH}$ 5.0 to $\mathrm{pH} 5.6$ are presented in Table 1 . The turbidity analysis provides the final operating $\mathrm{pH}$ and wall ratio for complex coacervation of CO using Chitosan-Gelatin (B) system. Similar finding with [25] work was obtained where initially both Chi and Gel-B solutions appeared almost transparent in colour when they are alone as solutions. The transparency decreased when these two polymers were mixed together forming turbid or cloudy mixture as $\mathrm{pH}$ increased. In this case, turbidity represents the formation of insoluble complexes and causing increase in absorbance [24]. Since transmittance having opposite relationship with concentration and absorbance, thus when transmittance is low, the turbidity will be high [32]. Fig. 3 represents the percent transmittance data for Chitosan-Gelatin (B) mixture against $\mathrm{pH}$. Based on this figure, it shows that as $\mathrm{pH}$ increased, the percent transmittance of Chitosan-Gelatin (B) mixture was decreased resulting increment in turbidity. According to [33], charges on the polyelectrolytes must be sufficiently large to cause significant electrostatic interactions for complex coacervation to occur, but too large charges can cause precipitation. In selecting best wall ratio and 
operating $\mathrm{pH}$, condition where Chitosan-Gelatin (B) mixture become precipitate need to be avoided. In this study, we observed that Chitosan-Gelatin (B) mixture at $\mathrm{pH} 5.4$ and 5.6 showed remarkable precipitation. Therefore, only $\mathrm{pH} 5.0$ and $\mathrm{pH} 5.2$ left for the selection of final operating $\mathrm{pH}$ and wall ratio. Based on the turbidity analysis done by [25], the operating wall ratio was selected by taking the maximum turbidity or highest percent transmittance of chitosan and gelatin-B mixture which was at ratio 1:15. The maximum percent transmittance was observed at $\mathrm{pH} 5.0$ when the ratio of chitosan to gelatin was $1: 35$. Therefore, the operating process condition was set at wall ratio of 1:35 and $\mathrm{pH} 5$. Comparable operating condition was applied by [19], which emulsified the oil phase on the mixture of polymers (gelatin-B and chitosan) at $\mathrm{pH}$ 5.0.

\subsection{FT-IR spectra}

Analysis on the changes in chemical structures of each CO capsules was done by using FT-IR. The FT-IR spectra obtained at each process stage of $\mathrm{CO}$ encapsulation process are represented in Fig. 4. FT-IR spectra at emulsification stage is shown in Fig. 4(a) represent for $\mathrm{CO}$, Gel-B at $\mathrm{pH} 5$ and emulsion of CO+Gel-B. CO showed strong sharp peak at 2923.03 $\mathrm{cm}^{-1}$ and $2853.69 \mathrm{~cm}^{-1}$ for C-H stretching of alkanes and methyl C-H bending of alkanes at $1455.88 \mathrm{~cm}^{-1}$ and $1374.54 \mathrm{~cm}^{-1}$. Peak at $1229.92 \mathrm{~cm}^{-1}$ and $1108.75 \mathrm{~cm}^{-1}$ represent $\mathrm{C}-\mathrm{O}$ stretch of alcohol and few peak observed at wavelength around $888.02 \mathrm{~cm}^{-1}, 824.53 \mathrm{~cm}^{-1}$ and $721.35 \mathrm{~cm}^{-1}$ for alkenyl $\mathrm{C}-\mathrm{H}$ bending. Since this study used Java CO from Cymbopogon winterianus, which is rich in monoterpene alcohol such as citronellal, geraniol and citronellol [10], therefore alchohol and alkanes peak appearance in FT-IR spectra proven the characteristic of CO. Gel-B spectra showed strong broad peak at 3309.73 $\mathrm{cm}^{-1}$ which represent normal polymeric O-H stretching and N-H stretching of amines and amides. Besides, Gel-B spectra also showed a broad weak peak at $1737.90 \mathrm{~cm}^{-1}$ represent $\mathrm{C}-\mathrm{O}$ stretching of ester and a broad medium peak at $1635.37 \mathrm{~cm}^{-1}$ indicating $\mathrm{N}-\mathrm{H}$ bending of amines and C-O stretching of amides respectively. Methyl C-H bends appeared at peak $1365.92 \mathrm{~cm}^{-1}$ and a broad but weak peak at $1267.03 \mathrm{~cm}^{-1}$ for C-O stretching of ester group also indicating Gel-B characteristic. Spectra for emulsion of $\mathrm{CO}+\mathrm{Gel}-\mathrm{B}$ showed disappearance of all $\mathrm{CO}$ peaks left alone peak at $3308.51 \mathrm{~cm}^{-1}, 2108.80 \mathrm{~cm}^{-1}$ and $1635.40 \mathrm{~cm}^{-1}$ which refer to Gel-B peak without the presence of peak at $1737.90 \mathrm{~cm}^{-1}, 1365.92 \mathrm{~cm}^{-1}$ and $1217.03 \mathrm{~cm}^{-1}$. The disappearance of all $\mathrm{CO}$ peak and some of Gel-B peak explained that Gel-B had acted as polyanions from $\mathrm{C}-\mathrm{O}$ stretching of carbonyl group, and then coated the $\mathrm{CO}$ droplet during emulsification stage.

Coacervation stage FT-IR spectra was shown in Fig. 4(b) for spectra of emulsion of $\mathrm{CO}+\mathrm{Gel}-\mathrm{B}$, Chi at $\mathrm{pH} 5$ and spectra after addition of Chi. For $\mathrm{Chi}$ at $\mathrm{pH} \mathrm{5,} \mathrm{the}$ spectra showed certain similarity to Gel-B spectra as mentioned by [23], where a broad strong peak appeared at peak $3308.80 \mathrm{~cm}^{-1}$ showed normal polymeric O-H stretching and N-H stretching of amines and amides. A broad weak peak at $1737.53 \mathrm{~cm}^{-1}$ represent $\mathrm{C}-\mathrm{O}$ stretching of carbonyl group and a broad medium peak at $1636.09 \mathrm{~cm}^{-1}$ indicating N-H bending of amines and C-O stretching of amides in Chi structure. Peak at $1365.82 \mathrm{~cm}^{-}$ ${ }^{1}$ and $1217.00 \mathrm{~cm}^{-1}$ for $\mathrm{C}-\mathrm{O}$ stretching showed presence of cyclic ether group in Chi. FT-IR spectra for $\mathrm{CO}+\mathrm{Gel}-$ $\mathrm{B}+\mathrm{Chi}$ showed similar pattern with FT-IR spectra for $\mathrm{CO}+\mathrm{Gel}-\mathrm{B}$ after addition of Chi. This explained that no significance chemical changes happened at coacervation stage. However in adjust $\mathrm{pH}$ stage shown in Fig. 4(c), two sharp medium peak at $1365.89 \mathrm{~cm}^{-1}$ and $1217.01 \mathrm{~cm}^{-}$ 1 of cyclic ether $\mathrm{C}-\mathrm{O}$ stretch from $\mathrm{Chi}$ peak was noticeable showing the polyanionic-polycationic interaction between Gel-B and $\mathrm{Chi}$ to form polyelectrolyte complex of Chi-Gel (B). Moreover, there are weak peak noticed around carbonyl region at 1800 $\mathrm{cm}-1$ to $1700 \mathrm{~cm}-1$ which proven this interaction as according to [23] explanation. Finding from [22] FT-IR analysis (using transmittance mode) described that $\mathrm{C}=\mathrm{O}$ group of gelatin interacted with $\mathrm{N}-\mathrm{H}$ groups of chitosan resulting intensity increased to $\mathrm{N}-\mathrm{H}$ bending and decreased to $\mathrm{C}-\mathrm{O}$ stretching vibration. In this study, peak at $1635.57 \mathrm{~cm}^{-1}$ of $\mathrm{N}-\mathrm{H}$ bending showed decreased intensity of absorbance whereas at peak $1217.01 \mathrm{~cm}^{-1}$ of $\mathrm{C}-\mathrm{O}$ stretching showed increased intensity of absorbance. It seem that opposite result was obtained if compared to previous description, however similar finding was actually obtained since absorbance and transmittance unit for FT-IR analysis having opposite relationship.

In dilution stage represent in Fig. 4(d), the FT-IR spectra obtained showed indifference on peak observed from previous stage (adjust $\mathrm{pH}$ ), since addition of water only reducing the concentration of the mixture hence reducing the intensity of the spectra obtained without changing the chemical structure. It was explained in [34] that adding sufficient water is for the purpose of maintaining fluidity and ease of agitation after the addition polyanion or polycation (coacervation stage). There was a peak notable at $1737.59 \mathrm{~cm}^{-1}$ in cooling stage when comparing with previous stage of dilution. Based on Fig. 4(e), the peak was corresponding to C-O stretch of ester group from Gel-B. It was due to gelation of Gel$\mathrm{B}$ upon cooling at temperature $10^{\circ} \mathrm{C}$. As according to [35], gelatin solution with a concentration more than $1 \%$ will increase its viscosity drastically when cooled to $35-40^{\circ} \mathrm{C}$, and form a gel when cooled further. Jason and Kalota (1996) also supported that complex coacervation emulsion mixture need to be cooled to a temperature in the range of from $5^{\circ} \mathrm{C}$ to about $15^{\circ} \mathrm{C}$ in order for the gelatin to gel or solidify into microcapsules. Fig. 4(f) showed FT-IR spectra at crosslinking stage for mixture at cooling stage, GSE-OPC alone, and after the addition of crosslinking agent, GSE-OPC. Based on the figure, GSEOPC showed a broad strong peak at $3309.04 \mathrm{~cm}^{-1}$ for hydrogen bonded $\mathrm{O}-\mathrm{H}$ stretch of phenol and a broad weak peak at $1737.77 \mathrm{~cm}^{-1}$ for aromatics combination bands. A broad medium peak at $1634.84 \mathrm{~cm}^{-1}$ for aromatic $\mathrm{C}=\mathrm{C}$ bending, alkenyl $\mathrm{C}=\mathrm{C}$ stretching and $\mathrm{C}$ $\mathrm{C}=\mathrm{C}$ symmetric stretch of alkenes. Lastly a broad weak peak at $1365.83 \mathrm{~cm}^{-1}$ signify bending of phenol $(\mathrm{O}-\mathrm{H})$ and alkanes $(\mathrm{C}-\mathrm{H})$, while a peak at $1217.15 \mathrm{~cm}^{-1}$ indicates C-O-C stretching of ether. FT-IR spectra obtained for 
GSE-OPC are tally with [36], where proanthocyanidins or condensed tannins are polyphenol compounds having complex chemical structure which the oligomers are joined by carbon-carbon bonds. The FT-IR spectra obtained after addition of GSE-OPC during crosslinking stage showed similar pattern to GSE-OPC spectra, indicated that GSE-OPC had coated the cooled capsules droplet of Chitosan-Gelatin (B). This result aligned with [37] which explained that if the oligomers of polyphenol are insoluble in the droplets (which refer to capsules); a membrane is formed around them thus encapsulating the droplet. The polyphenolic compound constitutes the droplet, and the crosslinking reaction stabilizes the molecule which was proven by establishment of ester bonds detected from infrared spectroscopy. Finding from FT-IR spectra in crosslinking stage also confirmed that the decreasing of absorbance intensity at $1635.26 \mathrm{~cm}^{-1}$ of $\mathrm{N}-\mathrm{H}$ bend and increasing of absorbance intensity at $1737.92 \mathrm{~cm}^{-1}$ of $\mathrm{C}-\mathrm{O}$ stretch of ester group that characterized Gel-B was due to crosslinking reaction from polyphenol element in GSE-OPC. FT-IR spectra in harvesting stage which presented in Fig. 4(g) shows no changes compared to previous stage (crosslinking) since harvesting stage just adding few more of water to give extra fluidity hence promotes better settling and separation process of the $\mathrm{CO}$ capsules.
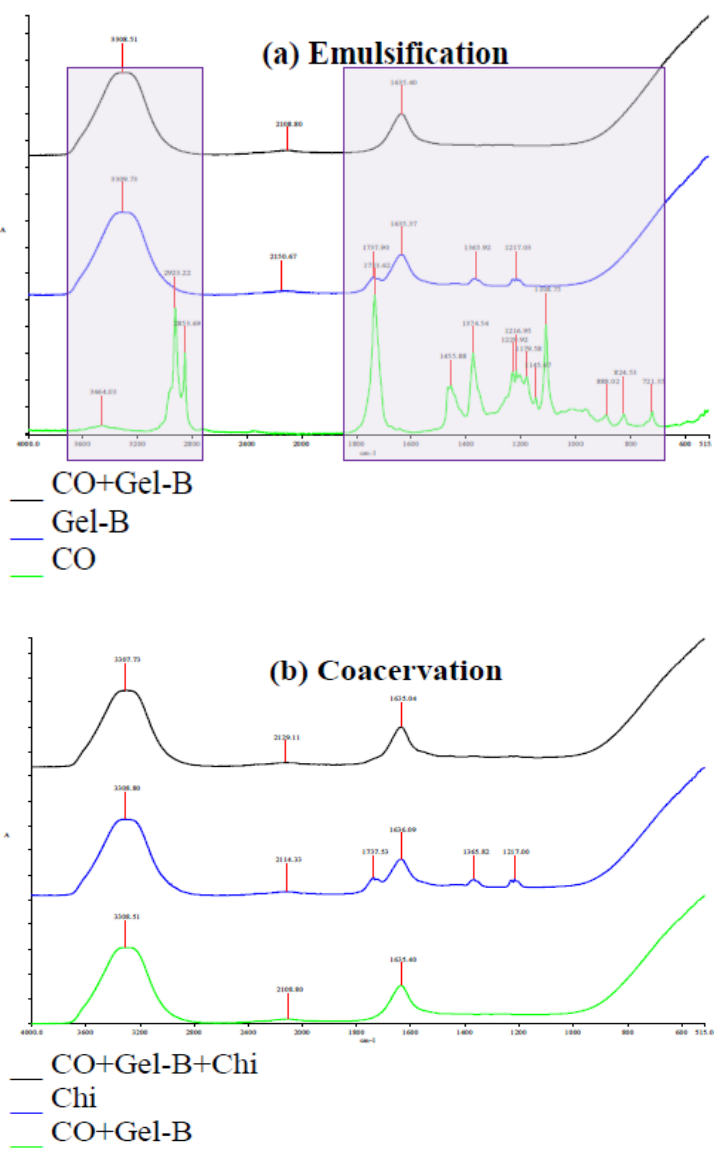

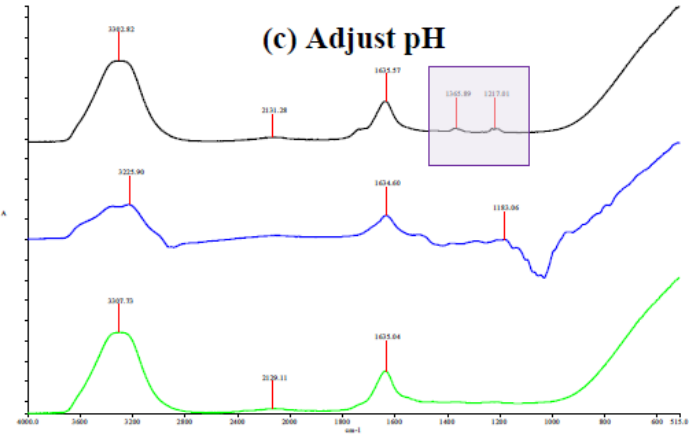

$\mathrm{CO}+\mathrm{Gel}-\mathrm{B}+\mathrm{Chi}(\mathrm{pH}$ )

$-\mathrm{NaOH}$

CO+Gel-B+Chi

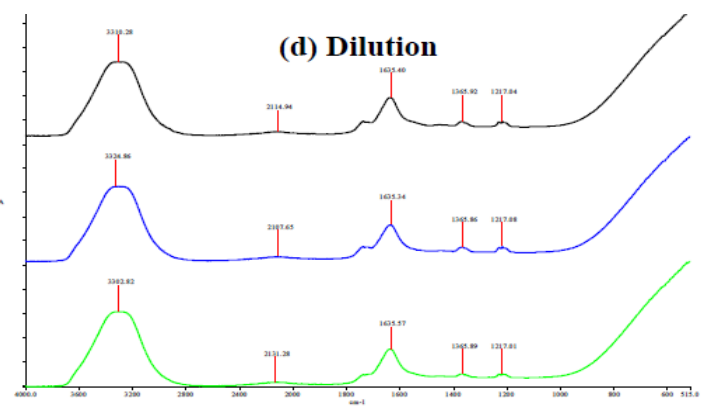

$\mathrm{CO}+\mathrm{Gel}-\mathrm{B}+\mathrm{Chi}(\mathrm{pH}$ 5) dilute

$\operatorname{DIW}\left(30^{\circ} \mathrm{C}\right)$

CO+Gel-B+Chi (pH 5)

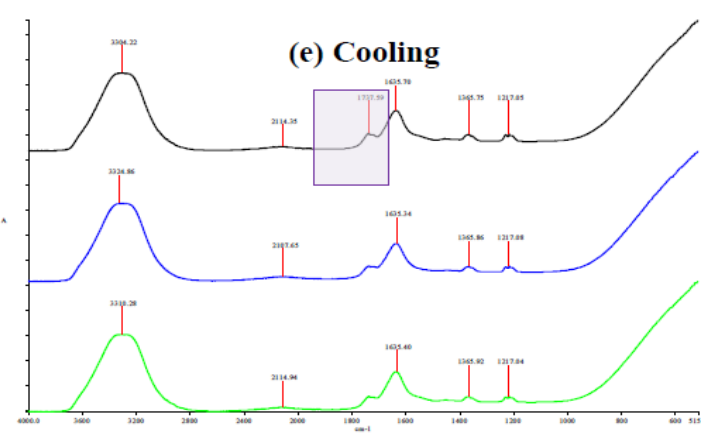

$\mathrm{CO}+\mathrm{Gel}-\mathrm{B}+\mathrm{Chi}(\mathrm{pH} 5)$ dilute $\left(10^{\circ} \mathrm{C}\right)$

$\operatorname{DIW}\left(10^{\circ} \mathrm{C}\right)$

CO+Gel-B+Chi (pH 5) dilute

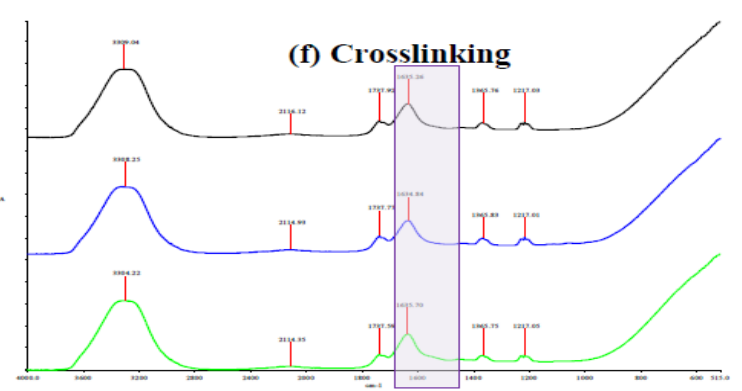

$\mathrm{CO}+\mathrm{Gel}-\mathrm{B}+\mathrm{Chi}(\mathrm{pH} 5)$ dilute $\left(10^{\circ} \mathrm{C}\right)+\mathrm{GSE}-\mathrm{OPC}$ GSE-OPC

$\mathrm{CO}+\mathrm{Gel}-\mathrm{B}+\mathrm{Chi}(\mathrm{pH} 5)$ dilute $\left(10^{\circ} \mathrm{C}\right)$ 


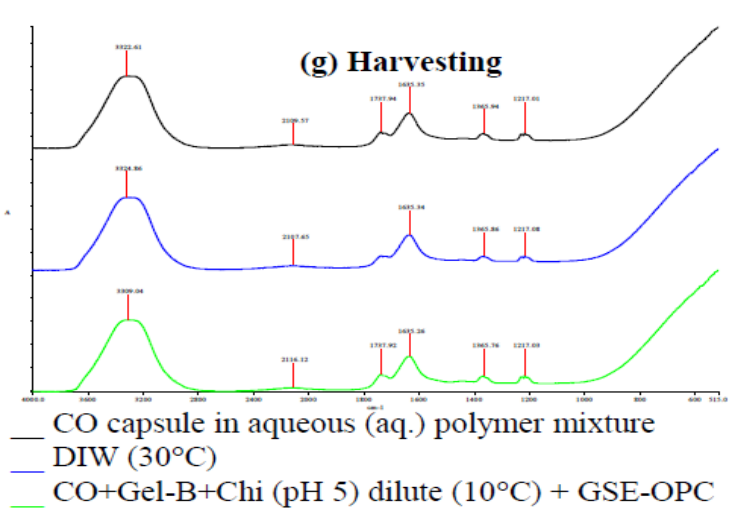

Figure 4. FT-IR spectra for each Citronella oil (CO) encapsulation process from (a) to (g).

Fig. 5 showed FT-IR spectra obtained at CO capsules collection process after settling process for more than 12 hours. Comparing each FT-IR spectra in Fig. 5, we distinguished that each spectra at top, middle and bottom layer showed quite similar pattern to spectra during harvesting stage. However, there are few significant peak noticed in top layer spectra at peak $2924.09 \mathrm{~cm}^{-1}$ for alkanes C-H strech, alkanes C-H bend at peak 1456.16 $\mathrm{cm}^{-1}$ and alcohol C-O strech at peak $1229.53 \mathrm{~cm}^{-1}$ and $1108.82 \mathrm{~cm}^{-1}$ respectively. The alcohol and alkanes peak here indicates $\mathrm{CO}$ existence at top layer which might be came from the unencapsulated $\mathrm{CO}$ accumulated after settling process or from the release of $\mathrm{CO}$ capsules at top layer. Since the middle and bottom layer FT-IR spectra showed quite similar pattern to spectra during harvesting stage without any sign of CO characteristics, we can said that middle layer consist of excess polymer with lots of water (dilute) and bottom layer contain concentrate excess polymer due to settling process. This finding was supported by [33], which explained that separation of the turbid reacted solutions in complex coacervation will produce two liquid phases, namely, the polymer-rich phase at the bottom and the supernatant at the top.

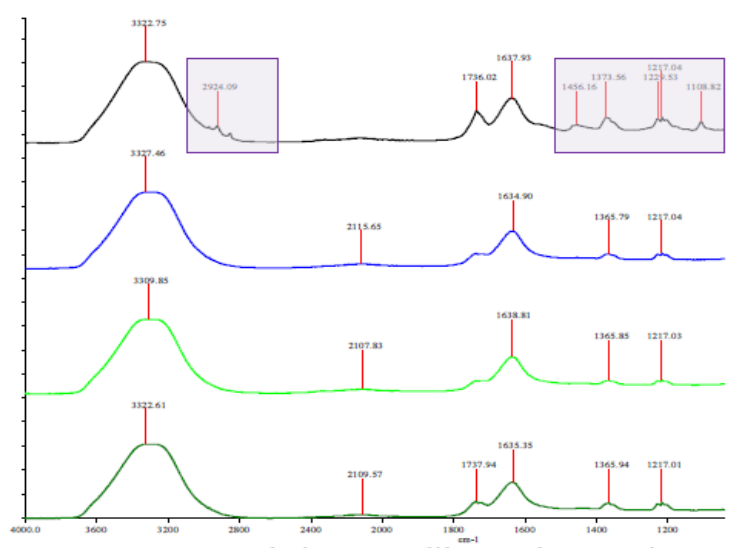

Top : CO capsule in super dilute polymer mixture Middle : dilute excess polymer mixture

Bottom : concentrate excess polymer mixture

_ CO capsule in aqueous (aq.) polymer mixture

Figure 5. FT-IR spectra at Citronella oil (CO) capsules product collection process.

\subsection{Optical microscope image}

Fig. 6 shows the optical microscopic image of each process step of Citronella oil (CO) encapsulation conducted from (a) to (g). In emulsification process (a), packed of $\mathrm{CO}$ droplets were observed due to high stirring process within transparent appearance of Gel-B solution. Coacervation process (b), takes place when the polycation from Chi solution attract to polyanions from Gel-B which surrounding CO droplet. From Fig. 6(b), the capsules were observed having spherical shape and also in a packed surrounding. After adjusting $\mathrm{pH}$ of the process to $\mathrm{pH} 5$ in (c), it was observed that the capsules surface become smoother but a certain degree of agglomeration happens due to the packed process surrounding. After dilution process (d), single spherical shape capsules with smooth surface were observed. The agglomeration occurrence in this process become lower since addition of de-ionized water (DIW) dilute the process system and larger the process surrounding which separate the capsules from each other. Fig. 6(e) shows the capsule condition after cooling process. We claimed here that the wall capsules were hardened since the cooling process was conducted below the gelation temperature of Gel-B which is at $10^{\circ} \mathrm{C}$. In crosslinking process (f), there are no major changes in surface morphplogy were observed. The final process of harvesting ( $g$ ) shows that the capsules are in single spherical shape and having smooth surface. In agreement with [14] based on observation obtained from optical micrograph, gelatin microcapsule appeared to be in round shape with relatively smooth surface. This statement supports earlier observation of $\mathrm{CO}$ harvesting process $(\mathrm{g})$, since the operating wall ratio in this study utilize higher amount of gelatin. Thus, it is possible for gelatin to dominate the physical properties of CO capsules obtained.

Fig. 7 shows microscopic images of Citronella oil (CO) capsules surface morphology at top layer after settling process We observed that the final products of $\mathrm{CO}$ capsules are in spherical shape which having irregular size. Some of the capsules were attached together causing agglomerate due to the hardened free gelatin-B after cooling until settling process. Agglomeration of capsules during wall formation was a common phenomenon in many microencapsulation processes [38]. As the wall materials change from liquid to solid form, they often went through a sticky stage which makes agglomeration difficult to be avoided [38]. From Fig. 7, we roughly described that the CO capsules obtained in this study are in micrometer size where the diameter varies from $81.63 \mu \mathrm{m}$ to $156.74 \mu \mathrm{m}$ with rough surface and almost spherical in shape. This result quite similar with [39] which obtained slightly rough surface yet close to spherical shape as a whole with average particle sizes was $294.7 \pm 14.2 \mu \mathrm{m}$ at emulsification stirring speed of $500 \mathrm{rpm}$. However, there is quite different where capsule produce in this study are much smaller in size, since [39] applied gelatin-arabic gum system as wall material. This statement was supported by [24] which claimed capsules produced by ChitosanGelatin (B) system (30 $\mu \mathrm{m}$ approximate diameter) are much smaller than those produced from Gelatin-Arabic 
gum system (100 $\mu \mathrm{m}$ approximate diameter). It might be due to the different in viscosity between these two types of complex system where Chitosan-Gelatin (B) system may be less viscous compared to Gelatin-Arabic gum system. Maji and Hussain (2009) revealed that increased in viscosity of the medium resulted in the formation of larger particle droplets during emulsification process. Less viscous material will increase shear rate thus produce smaller particle. This finding was supported by [40] that size of the coacervate suspensions decreased with decrease in viscosity of the material.

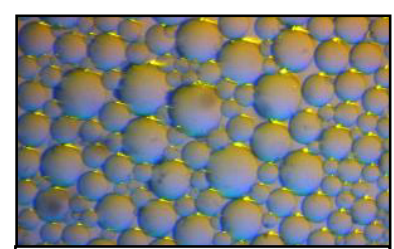

(a) Emulsification

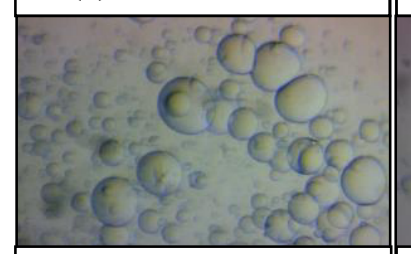

(c) Adjust pH
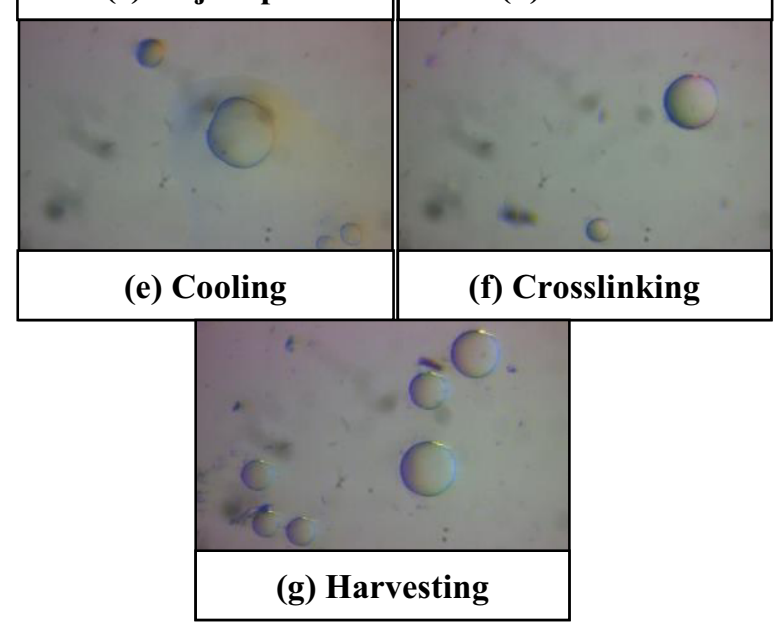

Figure 6. Microscopic image at $30 \mathrm{x}$ magnifications for each Citronella oil (CO) encapsulation process from (a) to $(\mathrm{g})$.

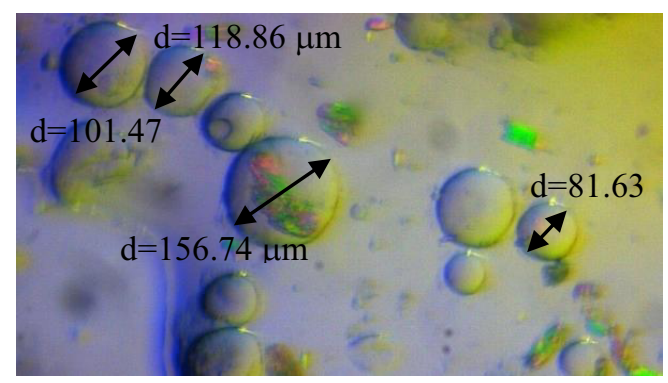

Figure 7. Microscopic images of Citronella oil (CO) capsules surface morphology at top layer after settling process under 30 $\mathrm{x}$ magnifications.

\section{Conclusions}

We found that zeta potential values for Chi acts as polycation within range of $\mathrm{pH} 4.0$ to 5.6, whereas Gel-B acts as polyanion above its isoelectric point (IEP) around $\mathrm{pH}$ 4.9. Wall ratio of $1: 35$ and $\mathrm{pH} 5$ was selected as design operating process for $\mathrm{CO}$ capsule production for this study. FT-IR analysis found that Gel-B had coated the $\mathrm{CO}$ droplet during emulsification stage, with Chi started to interact with Gel-B to form polyelectrolyte complex in adjust $\mathrm{pH}$ stage, $\mathrm{CO}$ capsules solidified at cooling process and were hardened by GSE-OPC layer during crosslinking process. Final product of $\mathrm{CO}$ capsules after settling process was identified at the top layer. The other middle and bottom layer were indicated as excess polymer with lots of water. Optical micrograph of $\mathrm{CO}$ capsules shows nearly round shape with quite smooth surface throughout each process stage. Final product of $\mathrm{CO}$ capsules obtained in this study can be described as having diameter varies from $81.63 \mu \mathrm{m}$ to $156.74 \mu \mathrm{m}$ with almost spherical in shape with rough surface. Low agglomeration was identified at the final process of $\mathrm{CO}$ capsules collection even though crosslinking stage that utilized GSE-OPC as the crosslinker was conducted as the counter measure. More study on crosslinking reaction and application of drying process after $\mathrm{CO}$ capsules collection could be suggested for future works. In this study, we can conclude that the proposed method of complex coacervation using chitosan-gelatin (B) system was successfully encapsulated the CO. Further investigation on $\mathrm{CO}$ capsules properties and performance will be carried out in the next study. We expect that this study will give better controlled release of $\mathrm{CO}$ which will further benefit industry that utilizes $\mathrm{CO}$ in their product.

\section{Acknowledgments}

The authors fully acknowledged Ministry of Higher Education (MOHE) Malaysia and Universiti Teknologi MARA Malaysia for the financial under research grants of 600-RMI/RAGS 5/3 (76/2013)

\section{References}

1. M.H. Hamzah, H. Che Man, H. Jamaludin, Z. Zainal Abidin, International Journal of Chemical Engineering and Applications, 3, 173-177 (2012)

2. N.A.M. Zin, R. Raslan, J. Jai, I. Subuki, $2^{\text {nd }}$ International Conference on Green Chemical Engineering Technology (GCET, 2015)

3. N.M. Ali, R. Raslan, J. Jai, I. Subuki, $28^{\text {th }}$ Symposium of Malaysian Chemical Engineers (SOMChe, 2015)

4. J. Mercola, Citronella oil: the all-natural bug repellent, Mercola.com. Retrieved on February 26, 2016 from http://articles.mercola.com/herbaloils/citronella-oil.aspx

5. R.E.D. Facts: oil of citronella, United State Environmental Protection Agency. Retrieved on $\begin{array}{llll}\text { February } & 26, & 2016 & \text { from }\end{array}$ 
http://www3.epa.gov/pesticides/chem_search/reg_act ions/reregistration/fs_PC-021901_1-Feb-97.pdf

6. C. Bond, K. Buhl, D. Stone, (2013), Oil of citronella: general fact sheet, National Pesticide Information Center, Oregon State University Extension Services. Retrieved on February 26, 2016 from http://npic.orst.edu/factsheets/citronellagen.pdf

7. B. Solomon, F.F. Sahle, T. Gebre-Mariam, K. Asres, R.H.H. Neubert, European Journal of Pharmaceutics and Biopharmaceutics, 80, 61-66 (2011)

8. M.A. Manaf, J. Jai, A.N. Mustapa, R. Raslan, I. Subuki, Applied Mechanics and Materials, 625, 860863 (2014)

9. U. Sakulku, O. Nuchuchua, N. Uawongyart, S. Puttipipatkhachorn, A. Soottitantawat, U. Ruktanonchai, International Journal of Pharmaceutics, 372, 105-111 (2008)

10. A. Wany, S. Jhaz, V.K. Nigam, D.M. Pandey, International Journal of Advanced Research, 1, 504521 (2013)

11. N. Rani, A. Wany, A.S. Vidyarthi, D.M. Pandey, Current Research in Microbiology and Biotechnology, 1, 98-103 (2013)

12. M.F. Maia, S.J. Moore, Malaria Journal, 10,1-15 (2011)

13. S.K. Chinta, P.W. Pooja, Indian Journal of Engineering, 3, 37-40 (2013)

14. S.Y. Cheng, M.C.W. Yuen, C.W. Kan, K.K.L. Cheuk, C.H. Chui, K.H. Lam, International Journal of Molecular Medicine, 24, 411-419 (2009)

15. F.R.A. Aziz, J. Jai, R. Raslan, I. Subuki, Advanced Material Research, 1113, 346-351 (2015)

16. L. Li, W. Au, T. Hua, D. Zhao, K. Wong, Textile Research Journal, 0, 1-6 (2013)

17. X. Jun-xia, Y. Hai-yan, Y. Jian, Food Chemistry, 125, 1267-1272 (2010)

18. D. Yu, W. Qiao, Q. Li, G. Pei, Journal of Fiber Bioengineering \& Informatics, 5, 67-76 (2012)

19. S.K. Basu, K. Kavitha, M. Rupeshkumar, Journal of Scientia Pharmaceutica, 78, 79-92 (2009)

20. C.A. Peptu, G. Buhus, M. Popa, A. Perichaud, D. Costin, Journal of Bioactive and Compatible Polymers, 25, 98-116 (2010)

21. M. Dennis and W. Brownrigg, "Development of micro-encapsulated phase change materials," Australia and New Zealand Solar Energy Society Conference (Solar 2006), ed. Conference Program Committee, Australian and New Zealand Solar
Energy Society (ANZSES), Canberra Australia, pp. 1-12, 2006.

22. S. Kim, M.E. Nimni, Z. Yang, B. Han, Wiley InterScience, 442-450 (2005)

23. T.K. Maji, M.R. Hussain, Journal of Applied Polymer Science, 111, 779-785 (2008)

24. A.S. Prata, C.R.F. Grosso, Carbohydrate Polymers, (2014)

25. M.K. Kang, J. Dai, J. Kim, Journal of Industrial and Engineering Chemistry, 18, 355-359 (2011)

26. H. Alavi Talab, M. Ardjmand, A.A. Motallebi, R. Pourgholam, Iranian Journal of Fisheries Sciences, 9, 199-208 (2009)

27. M.M.M. Specos, G. Escobar, P. Marino, C. Puggia, M. Victoria, D. Tesoriero, L. Hermida, Journal of Industrial Textiles, 40, 13-32 (2010)

28. L.F. Siow, C.S. Ong, Journal of Food Processing \& Technology, 4, 1-5 (2012)

29. M.E.I. Badawy, E.I. Rabea, International Journal of Carbohydrate Chemistry, 2011, 1-29 (2011)

30. M.C. Silva, C.T. Andrade, Polímeros: Ciência e Tecnologia, 19, 1-5 (2009)

31. K.E. Patrick, S. Abbas, Y. Lv, I.S.B. Ntsama, X. Zhang, Pakistan Journal of Food Sciences, 23, 17-25 (2013)

32. R.T. Balch, ANALYTICAL EDITION, 3, 124-125 (1931)

33. H.B. Bohidar, J. Surface Sci. Technol., 24, 105-124 (2008)

34. M.E. Jason, D.J. Kalota, U.S. Patent 5540 927, (1996)

35. S.N. Molnes, Physical properties of gelatin based solid emulsions: effects on drug release in the GI tract, M.S. thesis, Dept. of Biotechnology, Norwegian Univ. of Science and Technology, Trondheim, (2013)

36. N. Tazzini, (2014), Proanthocyanidins: definition, structure and absorption, Tuscany Diet. Retrieved on March 4, 2016 from http://www.tuscanydiet.net/2014/02/12/proanthocyanidins-definitionstructure-absorption/

37. A. Munin and F.E. Lévy, Pharmaceutics, 3, 793-829 (2011)

38. H. Nack, J. Soc. Cosmetic Chemists, 21, 85-98 (1970)

39. C.P. Chang, T.K. Leung, S.M. Lin, C.C. Hsu, Colloids and SurfacesB: Biointerfaces, 50, 136-140 (2006)

40. E. Kizilay, A.B. Kayitmazer, P.L. Dubin, Advances in Colloid and Interface Science, 167, 24-37 (2011) 